\title{
Delayed Presentation of a Congenital Cholesteatoma in a 64-year-old Man: Case Report and Review of the Literature
}

\author{
${ }^{1}$ Department of Ear, Nose and Throat, Royal Perth Hospital, Perth, \\ Western Australia, Australia \\ 2 Department of Otolaryngology, Princess Margaret Hospital, Perth, \\ Western Australia, Australia \\ ${ }^{3}$ Department of Otolaryngology, Head and Neck Surgery, Fremantle \\ Hospital, Perth, Western Australia, Australia \\ ${ }^{4}$ Department of Otolaryngology, University of Western Australia, \\ Crawley, Western Australia, Australia
}

Nishant Davidoss ${ }^{1}$ Jennifer $\mathrm{Ha}^{2}$ Rupan Banga ${ }^{3}$ Gunesh Rajan ${ }^{4}$

\author{
Address for correspondence Nishant Davidoss, MBBS (Hons), \\ Department of Ear, Nose and Throat, Royal Perth Hospital, Wellington \\ Street Campus, 197 Wellington Street, Perth, WA 6000, Australia \\ (e-mail: Nishant.Davidoss@health.wa.gov.au).
}

J Neurol Surg Rep 2014;75:e113-e116.

\begin{abstract}
Keywords

- cholesteatoma

- congenital

- mastoid

- massive

- large

- incidental

- hearing loss

- management

- review

Introduction Congenital cholesteatomas of the temporal bone are epidermoid cysts of embryologic origin that result in progressive desquamation and trapping of squamous epithelium behind an intact tympanic membrane. They are benign, slowly progressive lesions that can be found in various areas of the temporal bone. We report a case of a patient with a massive cholesteatoma first detected at the age of 64 years, causing significant destruction of the mastoid and petrous temporal bones, and adjacent occipital bone.

Methods We reviewed the literature and a case report of a patient seen in our institution recently. The Medline database was used to search multiple terms including "congenital" and "cholesteatoma."

Results The patient's congenital cholesteatoma was detected incidentally on a computed tomography scan when the patient's only symptoms were unilateral conductive hearing loss with a family history of hearing loss. It was subsequently successfully operated on with minimal postoperative complications.

Conclusions Congenital cholesteatomas of mastoid origin can often exist for many years in a subclinical state and develop into a massive size before causing symptoms. A high index of suspicion is necessary to detect congenital cholesteatomas in patients with unilateral conductive hearing loss who are otherwise asymptomatic and have a normal tympanic membrane.
\end{abstract}

\section{Introduction}

Congenital cholesteatomas of the temporal bone are epidermoid cysts of embryologic origin that result in progressive desquamation and trapping of squamous epithelium behind an intact tympanic membrane. ${ }^{1}$ They are benign, slowly progressive lesions that can be found in various areas of the temporal bone. ${ }^{1}$ We report a case of a patient with a massive cholesteatoma first detected at the age of 64 years, causing significant destruction of the mastoid and petrous temporal bones, and adjacent occipital bone. received

August 23, 2013 accepted after revision

March 15, 2014

published online

June 24, 2014
DOI http://dx.doi.org/

10.1055/s-0034-1376200. ISSN 2193-6358.
@ 2014 Georg Thieme Verlag KG
Stuttgart · New York

License terms

(®) $\Theta \circledast$ 


\section{Method}

A literature search was performed with the keywords "cholesteatoma," “congenital," "mastoid," "massive," "large," “incidental," "hearing loss," "management," and "review" from Medline. The data were evaluated for relevance, currency, and quality before being reviewed.

\section{Case Report}

A 64-year-old mam presented with a 6-month history of mild hearing loss of the left ear. There was no history of otorrhea, tinnitus, vertigo, or pain. On examination, two areas of bony dehiscence over his mastoid and occiput were found. Otoscopy revealed an intact tympanic membrane with a yellow mass in the mesotympanum. Examination of cranial nerves was unremarkable. Audiometry demonstrated mild to moderate mixed hearing loss with reduced middle ear compliance. Temporal bone computed tomography (CT) showed an extensive soft tissue mass involving the left middle ear cavity, the left mastoid and petrous bones, as well as the occipital bone with mild compression of the cerebellum of the posterior fossa on that side ( - Fig. 1). This was further investigated with magnetic resonance imaging (MRI), which confirmed the presence of a well-circumscribed mass in the left occipital bone with contiguous involvement of the mastoid and petrous temporal bones. Diffusion-weighted imaging (DWI) confirmed the diagnosis of a cholesteatoma or epidermoid cyst (-Figs. 2 and $\mathbf{3}$ ).

A postauricular approach was performed. Elevation of the soft tissue flaps revealed the cholesteatoma sac. Remnant bony bridges were removed, followed by initial debulking of the cholesteatoma. The cholesteatoma (-Fig. 4) was found extending up to the foramen magnum inferiorly, superiorly up to the middle fossa dura, and posteriorly onto the posterior fossa dura. Anteriorly, it extended into the middle ear. The middle

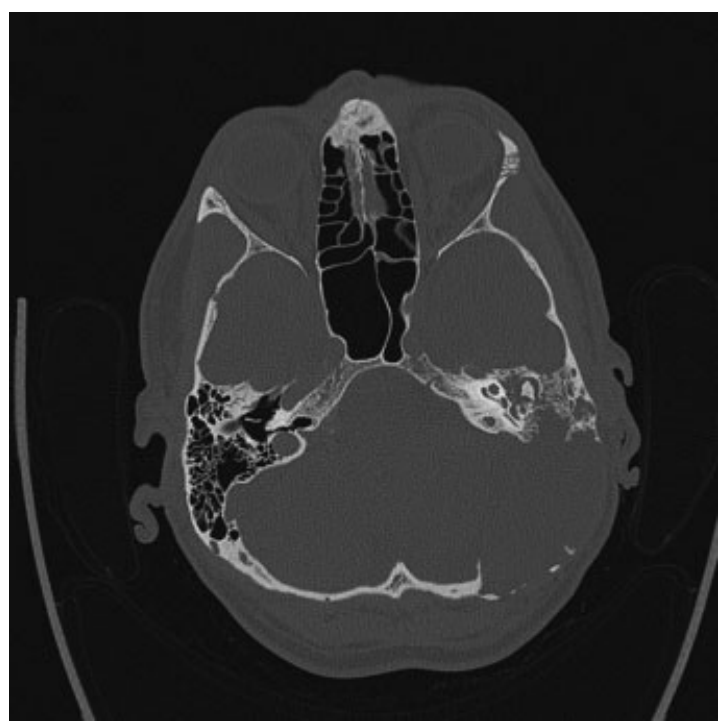

Fig. 1 Computed tomography scan of the temporal bone. Axial view demonstrating extra-axial left mastoid mass.

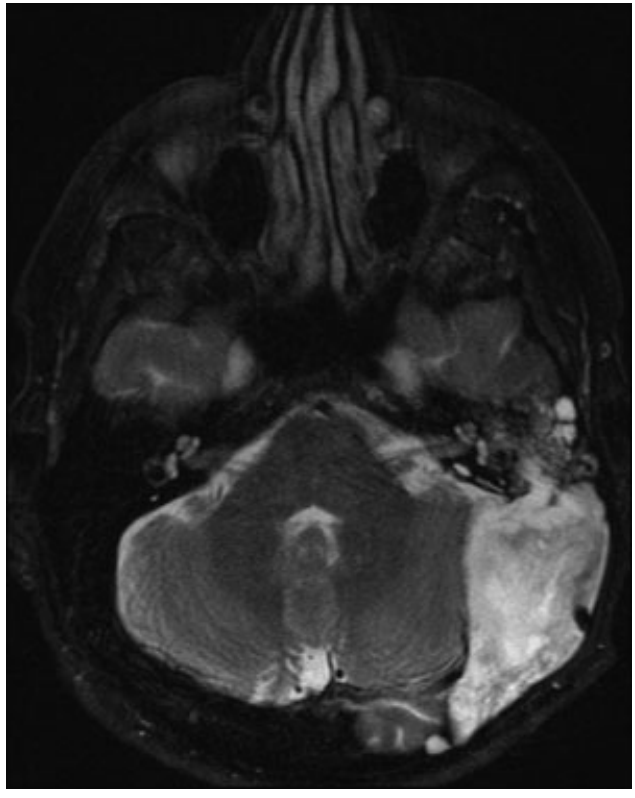

Fig. 2 Magnetic resonance imaging scan of the temporal bone. Axial view demonstrating giant mass eroding the mastoid and petrous temporal bones.

fossa and posterior fossa dura were exposed and the cholesteatoma matrix peeled off. The cholesteatoma was completely removed with a drill-out of the cholesteatoma cavity, removal of the mastoid tip, and clearance of the inferomedial extension into the occipital bone adjacent to the foramen magnum. The cavity was obliterated with abdominal fat. Histopathologic examination confirmed this lesion to be a cholesteatoma measuring $12.7 \times 8.9 \times 2.5 \mathrm{~cm}$. A 6-month follow-up MRI displayed a stable surgical resection bed that was disease free. Diffusion-weighted MRI also accurately showed two small intra-calvarial foci of residual disease that were out of the resection site pending further excision (-Fig. 5).

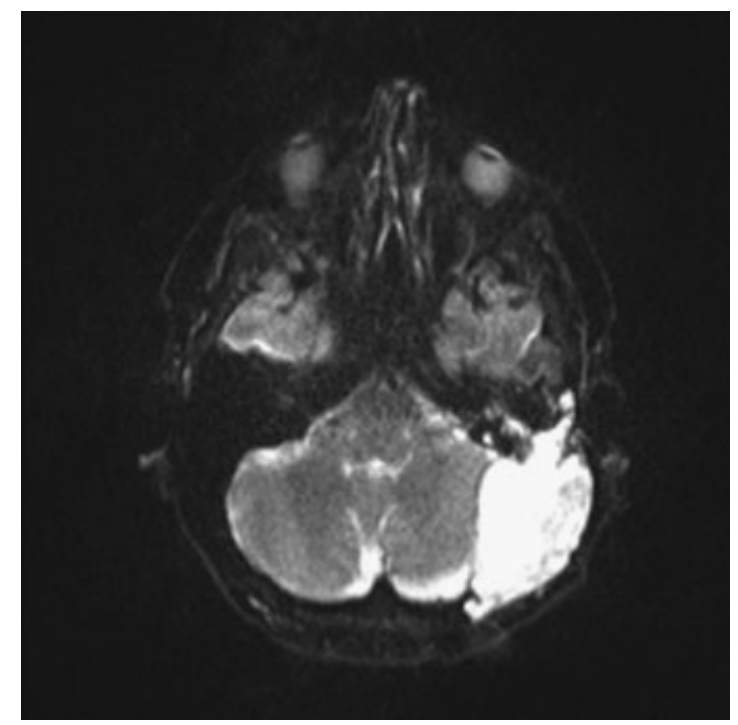

Fig. 3 Diffusion-weighted imaging of the temporal bone. Axial view demonstrating the presence of a cholesteatoma. 


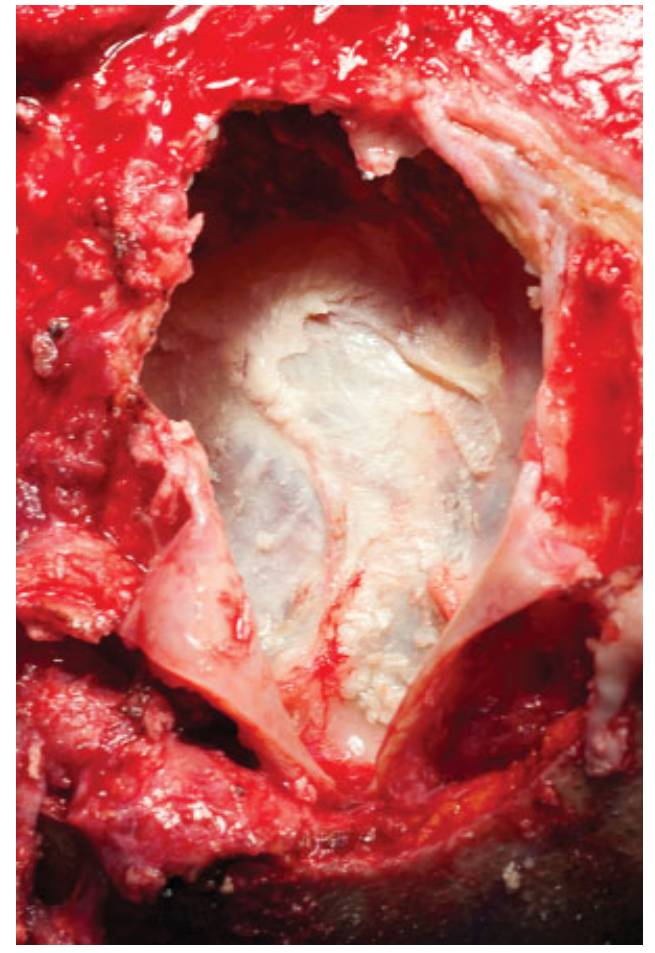

Fig. 4 Intraoperative finding of a large cholesteatoma sac with the matrix lining the posterior fossa dura.

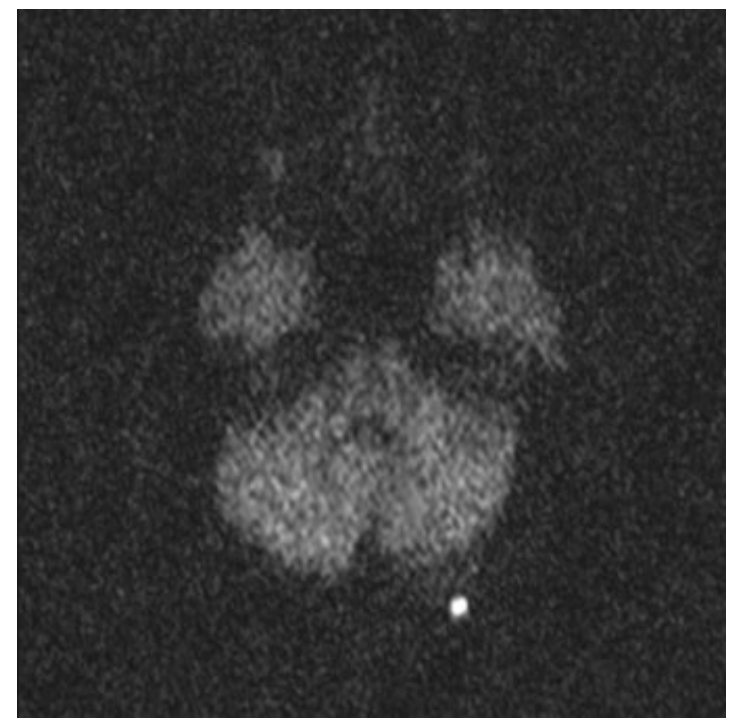

Fig. 5 Diffusion-weighted imaging of the temporal bone. Axial view demonstrating the presence of a focus of residual disease.

\section{Discussion}

Congenital cholesteatomas are epidermoid cysts that arise as a result of progressive desquamation of the epithelium. ${ }^{1}$ Unlike acquired cholesteatomas, they are often asymptomatic behind an intact tympanic membrane. ${ }^{2}$ As it was with this case, the first symptom is usually hearing loss. ${ }^{2}$

The underlying mechanism of development of congenital cholesteatomas remains largely unknown. ${ }^{3}$ However, the proposed mechanism for the development of congenital cholesteatomas is the failure of reabsorption of embryonic epidermoid residue that leads to the development of a cyst behind an intact tympanic membrane. ${ }^{3}$ They often arise from the anterosuperior quadrant of the mesotympanum and are often found to involve the mastoid, middle ear, and petrous bone. ${ }^{1}$ Because of how large the mass was in our patient, it was impossible to determine accurately the exact site of origin. Congenital cholesteatomas present differently depending on their location. ${ }^{1,4}$ Patients with congenital cholesteatomas in the middle ear often present with subtle hearing loss during childhood years, where routine otoscopy may pick up a cholesteatoma behind a tympanic membrane. ${ }^{1,4}$ However, congenital cholesteatomas may be completely asymptomatic, especially when arising in areas such as the mastoid where the diagnosis occurs only as an incidental finding on imaging. ${ }^{1,4}$ As a result, the diagnosis is often delayed until mid to late adulthood due to the silent nature of the disease. ${ }^{1,4}$ In such cases, the most common symptoms are dizziness, pain, or swelling that may be localized to the temporal area of the head or to the neck. ${ }^{1,4,5}$ As was the case with our patient, these lesions are also often much larger in cases where diagnosis is delayed until adulthood. . $^{2,5}$

The use of common imaging modalities such as highresolution CT and MRI are important to detect and also define the extent of the lesion, which is crucial for planning surgical intervention. ${ }^{1,3,4}$ CT scans confirm the presence and provide an indication of the size and extent of the lesion, and MRI helps further enhance the evaluation of lesions detected by distinguishing cholesteatomas from other soft tissue masses such as neuromas, schwannomas, or metastases. ${ }^{1,4}$ The use of DWI can help further distinguish cholesteatomas from other cystic masses. ${ }^{1,4}$

On reviewing the literature, there were very few cases documenting the presence of large cholesteatomas. The vast majority of the cases did not provide accurate sizes of the lesions. Previous to this, the largest lesion we found in the literature measured $8 \times 7 \times 3 \mathrm{~cm}$ and was an infected congenital cholesteatoma found in a 23-year-old woman. ${ }^{2}$

\section{Conclusion}

Massive congenital cholesteatomas are very rare, more so when they involve both the mastoid and petrous temporal bone; this case appears even more exceptional when the literature is critically reviewed. ${ }^{4}$ Congenital cholesteatomas of mastoid origin may grow silently over many years and develop into a massive size before being detected. ${ }^{5} \mathrm{~A}$ high index of suspicion is necessary to detect congenital cholesteatomas in patients with unilateral conductive hearing loss with a white mass behind an intact tympanic membrane. ${ }^{1,4}$

\section{References}

1 Bennett M, Warren F, Jackson GC, Kaylie D. Congenital cholesteatoma: theories, facts, and 53 patients. Otolaryngol Clin North Am 2006;39(6):1081-1094 
e116 Late Presentation of a Congenital Cholesteatoma Davidoss et al.

2 Rapoport PB, Di Francesco RC, Mion O, Bento RF. Huge congenital cholesteatoma simulating an intracranial abscess. Otolaryngol Head Neck Surg 2000;123(1 Pt 1):148-149

3 Nevoux J, Lenoir M, Roger G, Denoyelle F, Ducou Le Pointe H, Garabédian EN. Childhood cholesteatoma. Eur Ann Otorhinolaryngol Head Neck Dis 2010;127(4):143-150
4 Giannuzzi AL, Merkus P, Taibah A, Falcioni M. Congenital mastoid cholesteatoma: case series, definition, surgical key points, and literature review. Ann Otol Rhinol Laryngol 2011;120(11):700-706

5 Cvorovic L, Jovanovic MB, Milutinovic Z. Giant destructive congenital mastoid cholesteatoma with minimal clinical presentation. Otolaryngol Head Neck Surg 2011;144(5):821-822 